\title{
NUCLEAR MAGNETIC RESONANCE: ITS CLINICAL APPLICATION
}

\author{
R. E. STEINER
}

From the Royal Postgraduate Medical School, Hammersmith Hospital, London

Nuclear magnetic resonance (NMR) is a phenomenon in which particular atomic nuclei respond to the application of certain magnetic fields by absorbing or emitting electromagnetic radiation, a phenomenon which was first demonstrated by Bloch, Hansen and Packard in 1946. Its medical application was pioneered by Odeblad, a physicist from Sweden, during the 1950s and 1960s (Odeblad and Lindström 1955; Odeblad 1966). The first published NMR image was by Lauterbur in 1973 and subsequently groups of physicists in Nottingham and Aberdeen have developed further imaging systems. Live human images followed in the late 1970s and the first clinical images of the brain appeared in 1980.

At the present time a number of groups are involved in the clinical evaluation of NMR imaging in this country and the United States, and also in Germany and Holland. To date clinical experience is still small but the results so far suggest that NMR imaging will play a significant role in clinical diagnosis in the future.

Basic principles. Nuclear magnetic resonance has been widely used in analytical chemistry for the last few decades. The nuclei of some atoms behave like spinning magnets and when these atoms are exposed to static magnet fields their magnetisation is preferentially aligned in the direction of the static magnetic fields, producing a net nuclear magnetisation.

When these atoms are exposed to an additional magnetic field oscillating at the spin frequency of each nucleus, the nuclear magnetisation can be rotated from its original orientation through any given angle. Following this pertubation the magnetisation returns to its original position, during which time a small electrical signal can be detected in a surrounding coil. The rate at which magnetisation returns to its original position is described by two relaxation times, $T_{1}$ and $T_{2} . T_{1}$ describes recovery in the direction of the external static field and $T_{2}$ describes recovery perpendicular to this field. Relaxation in the direction of the magnetic field $\left(T_{1}\right)$ depends on interaction between protons and the surrounding nuclei and molecules, whereas relaxation in the transverse

Professor R. E. Steiner, $C B E$, MD, FRCR, FRCP

Department of Diagnostic Radiology, Royal Postgraduate Medical School, Hammersmith Hospital, Ducane Road, London W12 0HS, England.

(C) 1983 British Editorial Society of Bone and Joint Surgery $0301-620 \mathrm{X} / 83 / 5146 \$ 2.00$ direction $\left(T_{2}\right)$ depends on the interaction of nuclei with one another. Both $T_{1}$ and $T_{2}$ are sensitive indicators of the local molecular and nuclear environment. NMR scanning systems use sequences to produce images with varying dependence on these imaging indicators as well as on proton density.

There is no known hazard associated with NMR imaging as at present performed. Guidelines have been provided by the National Radiological Protection Board to avoid theoretical hazards due to heating effects and rapid switching of magnetic fields.

Imaging instrumentation. All NMR imaging machines are constructed around a large magnet which provides uniform static magnetic fields. Two systems are at present in use, an ordinary resistive magnet and a superconducting cryogenic system cooled with liquid helium and nitrogen. A more detailed account of imaging techniques has been published elsewhere by Pykett et al. (1982).

\section{Clinical application}

To date NMR imaging has been primarily focused on the examination of the brain, where high-level contrast between grey and white matter provides anatomical detail not available with other imaging techniques (Figs 1 and 2). Similarly images are also available in the sagittal (Fig. 3) and coronal planes and, unlike computerised tomography (CT), artefact from bone is no problem. A large variety of cerebral pathology has so far been investigated ranging from vascular diseases to inflammatory disorders and tumours (Bydder et al. 1982).

NMR images are also sensitive to flow and this can be manifested either by an increased signal from blood flowing into the slice or absence of a signal from blood flowing out of the slice. As a result it is possible to demonstrate occlusion to blood flow as well as abnormal blood flow in arterial malformation.

Demyelinating diseases such as multiple sclerosis have been of particular interest and so have examinations of infants and children where a high level of contrast between grey and white matter enables one to assess the progress of myelination. There is a rapid phase of myelination during the first two years of life followed by a slower phase continuing into the second decade (Johnson et al. 1983).

It is also possible to demonstrate the neural canal 


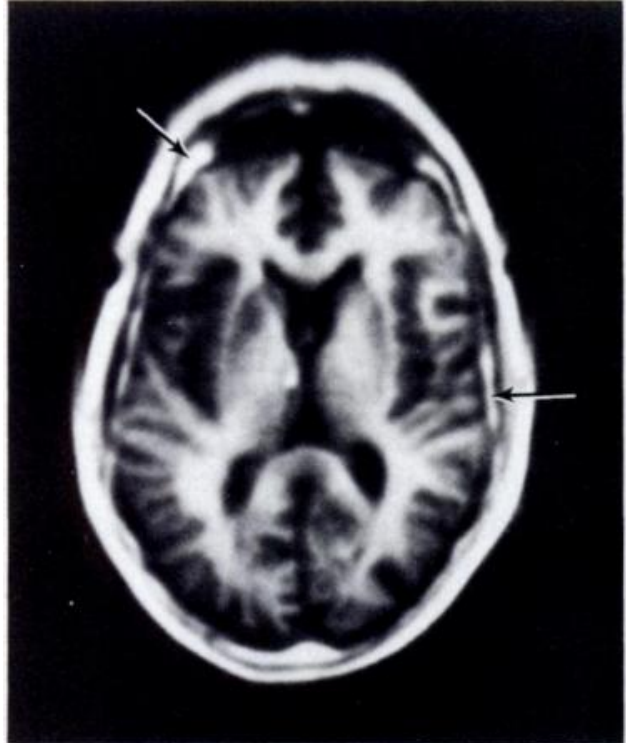

Fig. 1

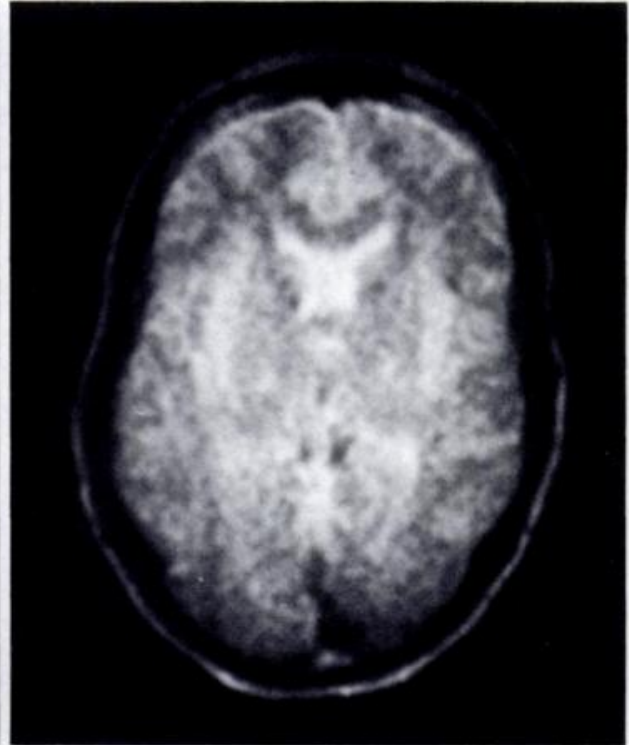

Fig. 2

Figure 1-Inversion recovery scan of a normal brain at the level of the ventricles. The white outer rim is due to subcutaneous fat with a short relaxation time. The black rim adjacent to it is the outer table of the calvarium with a very long relaxation time, which gives no signal. The arrowed white rim is the marrow in the diploic space. Note the clear demonstration of grey and white matter, the basal ganglia and black appearance of the cerebrospinal fluid with a very long relaxation time. Figure $2-$ Spin echo study on the same normal brain. There is hardly any differentiation between grey and white matter but the cerebrospinal fluid appears white due to its long relaxation time.

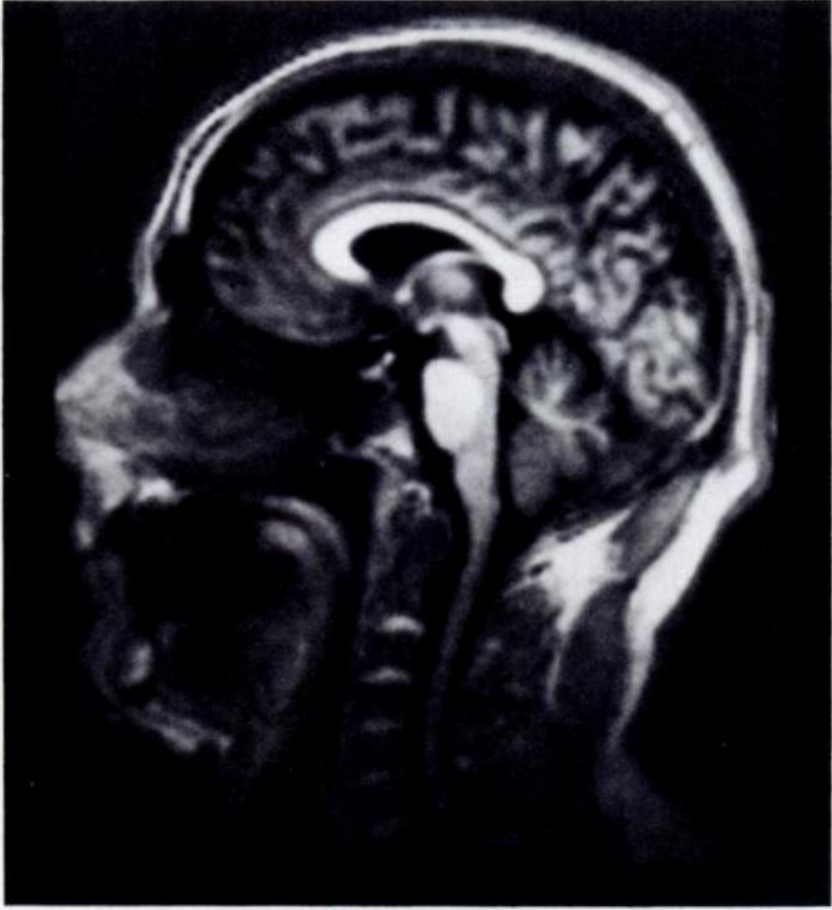

Fig. 3

Inversion recovery sagittal scan of the normal brain. This clearly demonstrates the hemisphere, the cerebellum, corpus callosum, mid brain and upper cervical cord as well as a great deal of other detail.

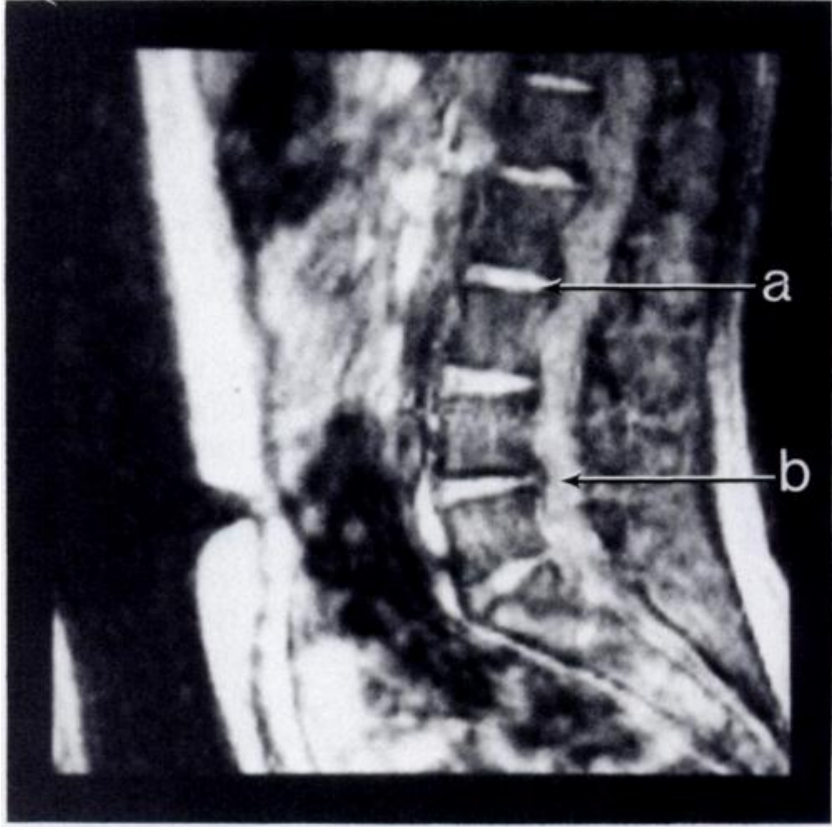

Fig. 4

Sagittal spin echo scan of the lower abdomen to demonstrate the vertebrae, intervertebral discs and the neural canal: $(a)$ intervertebral discs in white with a long relaxation time, $(b)$ the meningeal sac in the lower lumbar region. Note the vertebral bodies are visible due to the signal produced by the bone marrow and blood within. The bone itself does not give a signal and is therefore not visible. 


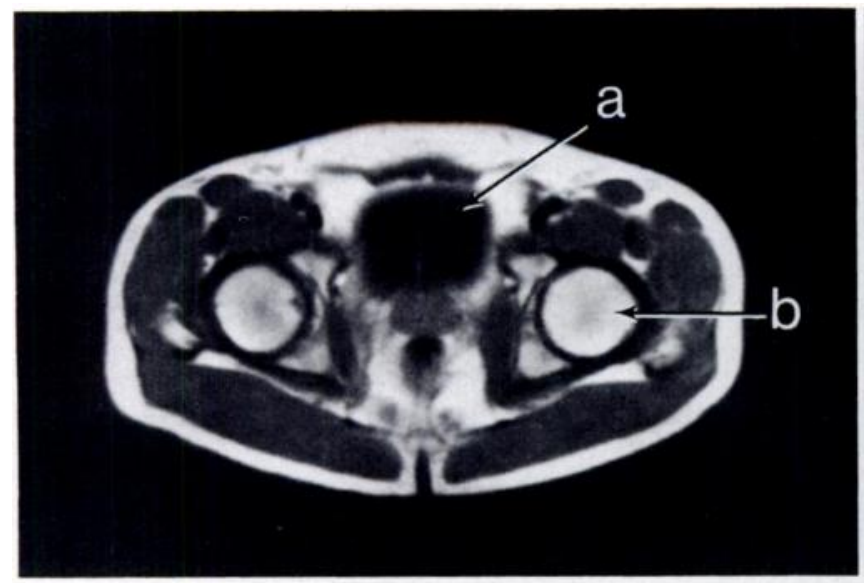

Fig. 5

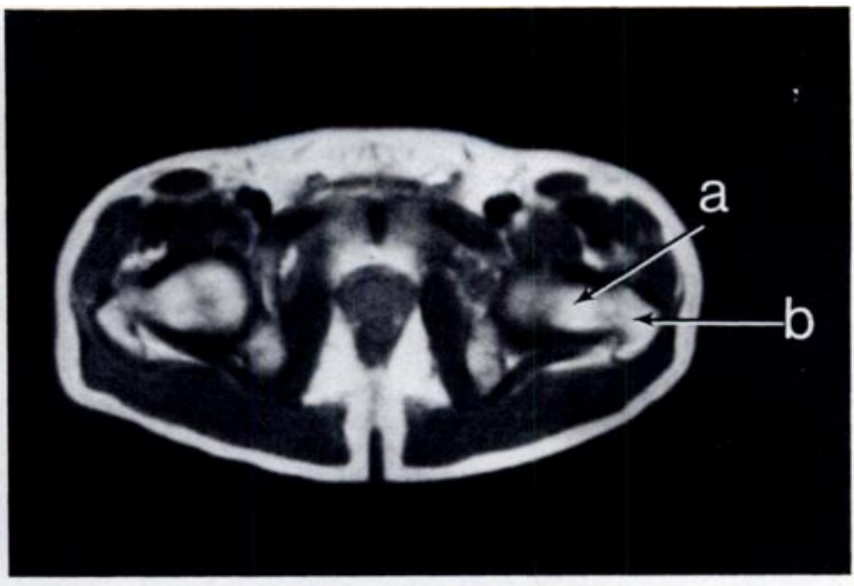

Fig. 6

Figure 5-High-resolution proton-density scan of a normal pelvis. The subcutaneous fat and pelvic fat are clearly visible in white, the muscle groups in grey and the septa between muscle groups in white due to fat. At the base of the bladder $(a)$ the prostate is visible and behind it the rectum. The femoral heads $(b)$ and the acetabula are well shown and so is the fovea on the left side and ligamentum teres on the right. Note also the thin rim and black centre of the femoral artery and vein in cross-section. Figure 6-A slice two centimetres lower in the same patient, which clearly shows the femoral neck $(a)$ and the greater trochanter $(b)$. The soft tissues are well demonstrated as on the previous slice.

and the cord within it, as well as the intervertebral discs and the nucleus pulposus(Fig. 4). This latter development may be of considerable interest to orthopaedic surgeons. This ability presents a significant advance and may in time partly replace myelography.

Clinical evaluation to date has also extended to softtissue structures of the thorax, such as the heart and mediastinum, and the large vessels (Steiner et al. 1983). Organs in the abdomen (the liver and pancreas) and in the retroperitoneal space (the kidneys and adrenal glands) are also being studied (Doyle et al. 1982).

Soft-tissue structures in the limbs, particularly muscle, are well demonstrated by this technique which appears to be superior to computerised tomography or even ordinary soft-tissue radiography. Not only normal muscle and surrounding soft tissues but also tumours and soft tissues in the region of the joints are well defined. In a recent communication Kean et al. (1983) demonstrated some of the muscle and fat structures in the knee joint.

Bone has very few unstable protons and therefore does not produce a NMR signal. Nevertheless, soft tissues associated with bone, such as marrow, blood vessels and the surrounding structures, muscle tendons and joint capsules, which do produce signals are very likely to make NMR imaging important and of interest to orthopaedic surgeons (Figs 5 and 6). To date experience in this particular area of study is still very limited; but in time, no doubt, sufficient experience will be gained and the application of NMR in the diagnosis of orthopaedic disorders will be evaluated and established.

\section{REFERENCES}

Bloch F, Hansen WW, Packard ME. The nuclear induction experiment. Phys Rev 1946;70:474-85.

Bydder GM, Steiner RE, Young IR et al. Clinical NMR imaging of the brain: 140 cases. AJR 1982;139:215-36.

Doyle FH, Pennock JM, Banks LM et al. Nuclear magnetic resonance imaging of the liver : initial experience. AJR 1982;138: $193-200$.

Hawkes RC, Holland GN, Moore WS, Worthington BS. Nuclear magnetic resonance (NMR) tomography of the brain: a preliminary clinical assessment with demonstration of pathology. J Comput Assist Tomogr 1980;4(5):577-86.

Johnson MA, Pennock JM, Bydder GM et al. Clinical NMR imaging of the brain in children. AJR 1983, in press.

Kean DM, Worthington BS, Preston BJ et al. Nuclear magnetic resonance imaging of the knee: examples of normal anatomy and pathology. Br $J$ Radiol $1983 ; 56: 355-64$.

Lauterbur PC. Image formation by induced local interactions: examples employing nuclear magnetic resonance. Nature 1973;242: 190-1.

Odeblad E, Lindström G. Some preliminary observations on proton magnetic resonance in biologic samples. Acta Radiol $1955 ; 43: 469-76$.

Odeblad E. Micro-NMR in high permanent magnetic fields: theoretical and experimental investigations with an application to the secretions from single glandular units in the human uterine cervix. Acta Obstet Gynecol Scand 1966;45 Suppl 2:1-188.

Pykett IL, Newhouse JH, Buonanno FS et al. Principles of nuclear magnetic resonance imaging. Radiology 1982; 143: $157-68$.

Steiner RE, Bydder GM, Selwyn A et al. NMR imaging of the heart : current status and future prospects. Br Heart $J 1983$, in press. 